2020-06-30

\title{
Moving Targets: Addressing Concept Drift in Supervised Models for Hacker Communication Detection
}

\author{
Susan McKeever \\ Technological University Dublin, susan.mckeever@tudublin.ie \\ Brian Keegan \\ Technological University Dublin, Brian.Keegan@tudublin.ie \\ andrei quieroz \\ Technological University Dublin, andrei.quieroz@tudublin.ie
}

Follow this and additional works at: https://arrow.tudublin.ie/scschcomcon

Part of the Robotics Commons

\section{Recommended Citation}

McKeever, S., Keegan, B. \& Quieroz, A. (2020) Moving Targets: Addressing Concept Drift in Supervised Models for Hacker Communication Detection, CyberSA 2020 : IEEE International Conference on Cyber Situational Awareness, Data Analytics and Assessment, Dublin, Ireland, June 2020

This Conference Paper is brought to you for free and open access by the School of Computer Sciences at ARROW@TU Dublin. It has been accepted for inclusion in Conference papers by an authorized administrator of ARROW@TUDublin. For more information, please contact arrow.admin@tudublin.ie, aisling.coyne@tudublin.ie,gerard.connolly@tudublin.ie.

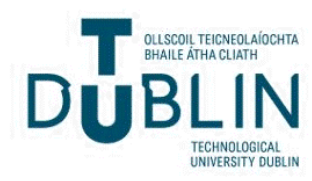




\section{Moving Targets: Addressing Concept Drift in Supervised Models for Hacker Communication Detection}

\author{
Andrei Lima Queiroz \\ Information Technology Secretariat - STI \\ University of Brasília - UnB \\ Brasília, Brazil \\ andreiqueiroz@unb.br
}

\author{
Brian Keegan \\ Applied Intelligence Research Centre \\ Technological University (TU) Dublin \\ Dublin, Ireland \\ brian.x.keegan@dit.ie
}

\author{
Susan Mckeever \\ Applied Intelligence Research Centre \\ Technological University (TU) Dublin \\ Dublin, Ireland \\ susan.mckeever@dit.ie
}

\begin{abstract}
In this paper, we are investigating the presence of concept drift in machine learning models for detection of hacker communications posted in social media and hacker forums. The supervised models in this experiment are analysed in terms of performance over time by different sources of data (Surface web and Deep web). Additionally, to simulate real-world situations, these models are evaluated using time-stamped messages from our datasets, posted over time on social media platforms. We have found that models applied to hacker forums (deep web) presents an accuracy deterioration in less than a 1-year period, whereas models applied to Twitter (surface web) have not shown a decrease in accuracy for the same period of time. The problem is alleviated by retraining the model with new instances (and applying weights) in order to reduce the effects of concept drift. While our results indicated that performance degradation due to concept drift is avoided by $\mathbf{5 0 \%}$ relabelling, which is challenging in real-world scenarios, our work paves the way to more targeted concept drift solutions to reduce the re-training tasks.

Index Terms-Cyber Security, Machine Learning, Concept Drift, Hacker Communication, Software Vulnerabilities
\end{abstract}

\section{INTRODUCTION}

In recent times, machine learning algorithms have been popularised due to their capacity for solving real-world problems through the discovery of useful patterns in data. These algorithms have revolutionised tasks such as image recognition (e.g., handwriting and face classification) and natural language understanding (e.g., sentiment analysis, Named-entity recognition). The enthusiasm surrounding data-driven learning approaches has expanded to a wide range of domains, including cyber security [1].

In general, machine learning models applied to cyber security tasks are designed with the assumption of a static world view. Such an assumption does not accommodate dynamic changes which present themselves over a period of time [2]. As a result, the accuracy of these models can be affected by changes in the problem concept, rendering the models less effective or useless as time goes on. This phenomenon is known as Concept drift and it is commonly seen in natural language tasks where the language usage and meaning changes constantly.
In cyber security initiatives that use hacker communications, movement in the concept is observed when new forms of malicious hacking emerge and are shared in social media channels. As seen in [3], new cyber related vocabulary (i.e. jargon) is created by hackers to communicate well-known security issues in computer systems. These forms of expression amongst peers are not static and are susceptible to changes. As seen in [4], between 2013 to 2017, there was an increase of new types vulnerabilities in software that were not previously categorised. This implies that hackers are communicating this type information by using different words and grammatical structures for expressing new concepts. It is in this dynamic context that cyber defenders are trying to operate to protect computer systems from threats.

For this reason, the aim of this paper is to investigate the extent and effect of concept drift on the accuracy of machine leaning (ML) models used to detect software vulnerabilities mentioned in social media posts. We base our experiment in the following research questions:

- Does the accuracy of our machine learning models, created to detect software vulnerabilities in social media posts degrade over time, indicating concept drift?

- Can we alleviate this problem using a typical concept drift solution method?

With this in mind, we carry out our work using 3 datasets, consisting of user posts gathered from social media and hacker forums. These datasets are gathered over an 8 year period, allowing a sufficiently long temporal window to observe and alleviate concept drift, if it exists. In order to visualise the concept drift, the messages which the models are trained and tested on are organised to consider the date and time where they were posted on social media platforms. We believe that this study can also be used as a base for detection of hacker communications regarding security problems in a new paradigms, such as Smart Cities and Internet of Things (IoT), which are constantly being shared in underground communities [5]

The structure of this paper is as follows: In Section 2, we 
review a selection of related works on software vulnerability communication detection and concept drift. In Section 3 we describe our approach, including a description of the datasets used and methodology. We also present our approach to quantify the effect of the concept drift in the models. In Section 4, we provide an analyses of the performance of the model in 1-year period for Twitter(surface web) and hacker forum (deep web) datasets. In section 5, we measure the slope of the line to identify the best approach to contain the deterioration of performance in these models. Finally, in Section 6, we summarise the contribution of this work and present the future work.

\section{RELATED WORK}

Recent work in the domain of hacker communication detection has shown little or no concern with the evolving nature (concept drift) of communications amongst peers. For instance, in [6], the authors provided an experiment using traditional classification algorithms to detect cyber discussions in social media channels. Also, in [7], the authors have provided a comparison of traditional Support Vector Machine (SVM) algorithm and Convolutional Neural Network (CNN) applied to the detection of hacker communications. Despite showing promising results, with high levels of classification accuracy, neither work considers the importance of the applicability and durability of their models over time under effects of concept drift.

To the best of our knowledge, there is no other work measuring concept drift in communication specific to software vulnerabilities in hacker forums and social media. However, our research has identified supporting work applied to the security domain, where the authors identified that the performance of machine learning models are affected by the rapidly evolving (drift) of malware software. As a result, further unseen malware is not identified by models trained in long time past data [8].

Other related studies on concept drift use specific algorithms for triggering the retraining of models when changes in the underlying distribution of the incoming data is detected. As seen in [9], the authors proposed an algorithm that observe the change in distribution of the data using dynamic sliding window. Furthermore, an algorithm proposed in [10] detects the drift in a on-line manner without storing datapoints in memory, which provides a more efficient algorithm in terms of memory resource. These algorithms applied to concept drift are of interest, as they indicate future options for our work.

\section{APPROACH}

\section{A. Dataset}

The 3 datasets used in this work have been used previously in [11] [12] and can be found in http://tiny.cc/8ws67y. They contain posts from on-line social media platforms such as forums and micro-blogs, commonly used by hackers for sharing and learning information about hacking and computer security in general. The subject of these messages range from technical to personal opinions and are related to a broad range of security topics, e.g., software security, data breach, copyright infringement, stolen credit card. Among these messages, we visually identify that few are related to malicious activities in software products or have mentioned security problems (flaws, vulnerabilities).

A summary description of each original dataset is described below:

D1 - CrackingArena Forum - This is a collection from one of the most used hacker forums existing in 2018 with 11,977 active users. It contains communications related to security issues in computing, which makes the data suitable for cyber security research on the interaction patterns among cyber criminals. The variety of covered topics in the forum ranges from social engineering, cracking/exploit tools to tutorials, which makes this forum a viable source for pinpointing the characteristics of newly emerged hacker assets.

D2 - Security Experts - The data contains posts from 12 security-expert users on Twitter, where six of them are part of the well-known-security experts having an average number of followers of 18,800, and the other six are part of the lesserknown security experts, having an average number of followers of 1,100 . Their tweets are mostly related to security aspects of technology, including software vulnerabilities and hacking.

D4 - Garage4Hackers Forum - This is a medium-sized forum in terms of number of content and users. This forum contains material related to exploitation, botnets and reverse engineering, it also provides information regarding specialised hacking tools.

The dataset D2 lies on the Surface Web domain as it is indexed by search-engines (e.g., google search) and can be freely accessed by every person with internet access. Whereas D1 and D4 lie on the domain of Deep web, which has its content accessed only by users with permission, credentials or invitation.

\section{B. Labelling Process}

The messages in these datasets were labelled consistently, using the following method. Each message was labelled 3 times, by three different computer science experts according to the following rules:

- Yes, for posts that appear as malicious communication regarding software vulnerabilities and exploitation.

- No, for posts that not appear as malicious communication regarding software vulnerabilities, which also include data breach, copyrighted software cracked, stolen accounts (e.g., Netflix, Amazon) and credit card accounts.

- Undecided, for messages where the labeller does not have enough information or confidence to mark as Yes or No.

The undecided choice is to cater for the ambiguity of certain posts, which is a non-trivial task (even for experts) to determine whether they are related to software security communication. The final label across the set of three labels was assigned according to the majority of votes. e.g., yes $=2$, no $=1$, undecided $=0$, the final label $(F L)=$ Yes. The posts with total disagreement between labellers, e.g., no $=1$, yes $=$ 
1 , undecided $=1$, were excluded from the dataset, which are less than $5 \%$ of the messages for all dataset.

In Table I, we have examples of messages and their respective labels. The message M1, marked as Yes, is related to a type of vulnerability (Stack Buffer Overflow) affecting a software product. Message M2, also marked as Yes, is related to a release of a Proof Of concept (PoC) of a vulnerability called dirtycow. The messages M3 and M4 are related to personal opinion and have no direct relation to real vulnerabilities in software. It is worth mentioning that despite M3 and M4 having hack and hacker keywords, they are not considered malicious.

\section{Transforming into a Binary Classification Task}

From a security standpoint, we believe it is prudent to capture the Undecided messages as a potential malicious problem. For this reason, we have transformed this task into binary classification. We have placed the Undecided and Yes messages into the positive class, whereas No messages are the negative class. Further details on the labelling task and description of social media platform used can be found in [12] [11].

It can be observed that all datasets have an imbalanced number of instances of positive and negative classes, with the minority proportion of these messages representing the positive, thus, malicious communication. In Table II, we present the description of each dataset with respect to the number of posts, the distribution of positive and negative instances and the average number of words.

In Fig 1, we present the volume of messages in each dataset over time. On the x-axis we have the year-month and on the $y$-axis the volume of posts collected during that period. The collected messages are spread in a period between 2010-07 and 2018-01 with each dataset covering a subset of this range of time. The dataset D1 contains messages between 2013-01 and 2018-01 (5 year), D2, the shortest dataset in terms of temporal range, contains messages between $2015-11$ and 2017-03 $(\approx 1$ year), D4, the longest, contains data between 2010-07 and 2017-13 ( $\approx 7$ year), To compare these experiments fairly we choose to use a 1-year peak for each, as the shortest dataset has about 1 year range.

\section{Experimental Methodology}

This experiment follows a stream-based methodology over a period of time. The following sections explains how the Predictive Sequential (Prequential) evaluation was performed over a Monthly Time Window (MTW) period. We also describe how we have trained the start model which proved to be the base for our experimental procedure.

1) Prequential Evaluation: Considering the changing nature of hacker language with the emergence of new forms of exploiting software vulnerabilities, we hypothesise that models trained in past instances will not present the same performance in new, or future, instances due the concept drift. For this reason, we propose to simulate the use of these models in reallife situations, where the model is trained and evaluated with consideration of the time when the messages occur in social media platform. The data used to feed the model is therefore organised by date of publication. For this streaming task, we are using Sliding Window prequential evaluation similar to [13], which in our case the windows are fixed by months of the year.

2) Monthly Time Window: In order to evaluate the performance of the model over a 1-year time period, we selected the use of a monthly time window to train and test the model. These time windows are organised for every 2 month period (bimester), although, for the training phase of the experiment, we have used 2 bimesters ( 4 months) due to the need of a minimum number of positive instances for applying oversampling technique as seen in [12]. This technique provides a performance enhancement of models built using imbalanced data. For the remainder of this work, the notation shown on the graphs are as follows: $w_{0}$, is the start model, which is trained and cross-validated in 2 bimesters messages, whereas the time window $w_{1}, w_{2}, w_{3}, w_{4}$ are the results of the test phase with the incoming messages with messages of 1 bimester each.

3) Training the Start Model: All experiments presented in this paper start with this model. The resulting performance is given by a 10-fold-cross validation and present in the graphs of this work at $w_{0}$ window. Considering that this model is trained with approximately $10 \%$ of instances from positive class (before resampling), we have applied the random oversampling technique to our training data as done in [12] to improve the classification performance of the model. The metric used to evaluate the model is the Average Class Accuracy Metric. This metric is the sum of the recall of the positive and negative classes divided by the number of classes as seen in (2) and recall being (1). This measure is commonly used to evaluate models trained on imbalanced datasets in order to capture class level accuracies. The performance achieved with the start model can be seen in Table III.

$$
\begin{aligned}
\text { Recall } & =\frac{\text { TruePositive }}{\text { TruePositive }+ \text { FalseNegative }} \\
\text { Avg.ClassAcc } & =\frac{\text { Recall }(\text { pos.Class })+\text { Recall(neg.Class })}{\text { No.Classes }}
\end{aligned}
$$

\section{EXPERIMENT - CONCEPT DRIFT IN 1-YEAR PERIOD}

In this experiment, we have selected D1, D2 and D4 as these datasets allow us to reach a minimum proportion of positive instances (roughly 10\%) for training the model within a 4month period. Thus, we can apply enhancement techniques (oversampling) to increase the performance of the model [12]. In addition, the source of these datasets differs on origin, as D1 and D4 are collected from hacker forums (deep web) and D2 is from Twitter (surface web).

For testing, we have used the remaining 8 months of messages. The evaluation was performed considering the time when the messages occur in social media platform. This was done to simulate real-life use of this model. The importance 


\begin{tabular}{lll}
\hline ID & Message & \\
\hline M1 & Multiple remote memory corruption vulns in all Symantec/Norton antivirus products, including stack buffer overflows & Yes \\
M2 & PoC for dirtycow vuln [URL] & Yes \\
M3 & Reading about lawyers argue about our Jeep hack is endless fun & No \\
M4 & it is amazing a hacker can put up with a sociologist ;) & No \\
M5 & Just released ssh_scan v0.0.10. Release notes can be found here & \\
M6 & I like sneaker's error 0xC0000156 & Undecided \\
\hline
\end{tabular}

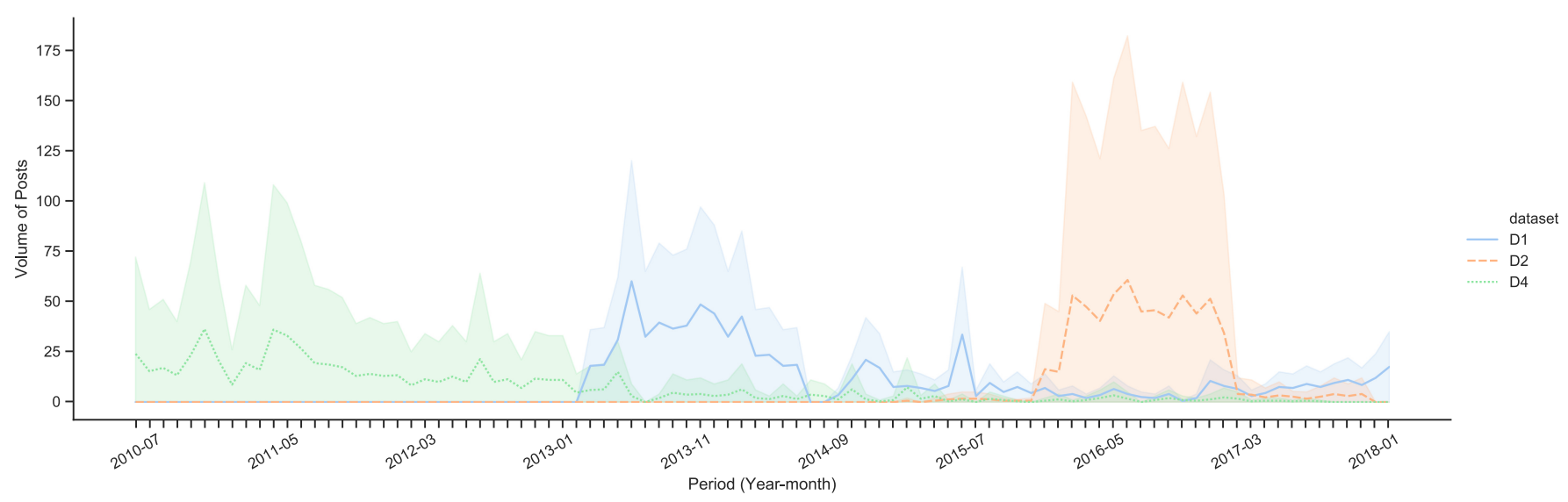

Fig. 1: Timeline and volume of messages of the datasets

TABLE II: DESCRIPTION DATASET

\begin{tabular}{lccccc}
\hline ID & Source & Type & $\begin{array}{c}\text { No. of } \\
\text { inst. }\end{array}$ & $\begin{array}{c}\text { Distrib. } \\
\text { (pos/neg) }\end{array}$ & $\begin{array}{c}\text { Avg. } \\
\text { words }\end{array}$ \\
\hline D1 & Hacker Forum & Deep web & 1,682 & $10 / 90 \%$ & 50 \\
D2 & Twitter & Surface web & 1,927 & $15 / 85 \%$ & 13 \\
D4 & Hacker Forum & Deep web & 1,966 & $13 / 87 \%$ & 78 \\
\hline
\end{tabular}

TABLE III: AVG. CLASS ACCURACY OF THE START MODEL (4 FIRST MONTHS)

\begin{tabular}{cc}
\hline & SVM+BOW \\
\hline D1 & 0.67 \\
D2 & 0.77 \\
D4 & 0.68 \\
\hline
\end{tabular}

of evaluating accordingly is to investigate how long the model would retain its performance after its creation - in other words, to measure the robustness of the model against the changes (drift) in the concept. Table IV presents the number of instances used in the training and test phase as well as the distribution of the classes in each month. The algorithm and feature representation used for this experiment are Support Vector Machine (SVM) with linear kernel and Bag-of-words (BOW) respectively. The SVM parameters are not tunned, instead, we used the library default values (Sci-kit 0.20.2v). We choose to use the defaults to fairly compare the performance of the models throughout all 3 datasets.

\section{A. Static Model Evaluation (Baseline)}

The purpose of this baseline experiment is to investigate the performance of static models over a period of 1-year. For this specific experiment, we are not performing any retraining (updating) of the model. We have used messages from a 4month period to train the model. In Table III, we see results of the training phase of this experiment. It is seen that, for hacker forums (D1 and D4), the model has achieved $67 \%$ and $68 \%$ of average class accuracy respectively, while Twitter source (D2) has achieved $77 \%$.

Moreover, Fig 2 presents the performance of the baseline model $(w 0)$ tested against messages of the remaining 8 months of the year $(w 1, w 2, w 3, w 4)$. We have constructed a regression line that best fits the data points over time. This line also represents the rise to run ratio of the model for each dataset. The $\mathrm{x}$-axis is the time window and the $y$-axis is the performance of the model in average class accuracy for that period of time. $w 0$ represents the results of the static model trained in 4 months and evaluated by 10 -fold cross validation. $w 1, w 2, w 3, w 4$ represent the results of the model tested in instances of a 2-month period.

Our baseline results show an apparent drift occurring in both hacker forums datasets (D1 and D4), whereas there is no apparent deterioration of the performance of model applied to D2 (Expert post on Twitter). We imply the hacker forums are environments that provide a more variate content than Twitter with regards to novelty on hacker techniques and tools, thus the drift on the former occurs earlier than in the latter.

Inspecting the absolute numbers, D1 starts at $61 \%$ avg. class acc. and ends in w4 with $59 \%$ of avg. class acc., whereas D4 has shown a bigger drift, starting at $68 \%$ and ending $56 \%$. Only D2 (twitter) has shown no sign of drift, with start and ending performance $77 \%$ avg. class acc, while the performance in $w 1, w 2, w 3, w 4$ does not decrease to less than the static 
TABLE IV: DISTRIBUTION OF INSTANCES IN EACH PHASE

\begin{tabular}{|c|c|c|c|c|c|c|c|c|}
\hline \multirow[b]{2}{*}{ Phase } & \multirow[b]{2}{*}{ Periods (months) } & \multirow[b]{2}{*}{ Window } & \multicolumn{2}{|c|}{ D1 } & \multicolumn{2}{|c|}{ D2 } & \multicolumn{2}{|c|}{ D4 } \\
\hline & & & No. Msgs & $\begin{array}{c}\text { Distrib. \% } \\
\text { (pos/neg) }\end{array}$ & No. Msgs & $\begin{array}{c}\text { Distrib. \% } \\
\text { (pos/neg) }\end{array}$ & No. Msgs & $\begin{array}{c}\text { Distrib. \% } \\
\text { (pos/neg) }\end{array}$ \\
\hline Train $^{1}$ & 1st to 4 th & w0 & 342 & $(33 / 67)$ & 826 & $(39 / 61)$ & 314 & $(44 / 56)$ \\
\hline Test & 5 th and 6 th & w1 & 144 & $(10 / 90)$ & 317 & $(11 / 89)$ & 179 & $(16 / 84)$ \\
\hline Test & 7 th and 8th & w2 & 149 & $(10 / 90)$ & 263 & $(13 / 87)$ & 89 & $(20 / 80)$ \\
\hline Test & 9th and 10th & w3 & 185 & $(12 / 88)$ & 291 & $(23 / 77)$ & 106 & $(7 / 93)$ \\
\hline Test & 11 th and 12 th & w4 & 150 & $(9 / 91)$ & 257 & $(19 / 81)$ & 207 & $(8 / 92)$ \\
\hline
\end{tabular}

${ }^{1}$ Positive class instances in training phase are oversampled by $300 \%$ according to [12]

model, in $w 0$, during the entire year.

\section{B. Dynamic Model Evaluation}

In this section, instead of using a static unchanging model, we present the results of a model retrained (updated) with incoming instances. We have also experimented with the introduction of weights on these new instances to increase the classification performance.

1) Updating Model with Past Instances: Differing from the static experiment, in this dynamic model we have performed a periodic update such that the model is tested in sample $P_{n}$ and outputs the average accuracy $W_{n}$, where $n \in\{1,2,3,4\}$ representing the periods of two months instances. Furthermore, the parameters of the model are updated considering the sample $P_{n}$. It is worth noting that there is no forgetting mechanism in this update process, which means that the old parameters still form part of the model. Fig 3 (b) shows a simplified graph of this procedure.

In order to investigate the minimum number of instances needed to update the model, and, thus, avoiding the decrease of performance over time, we have performed this experiment using different proportions of relabelled instances. The proportion are: 25\%, 50\%, 75\% and 100\% can be seen in Fig 4 . By the results, we see that, with all these proportions, the model has performed better than the baseline static model (thick line). It is also observed that, updating the model with $100 \%$ of the incoming instances gives, as expected, the best results. However, we can also reduce the update to $50 \%$ of the incoming instances and still have comparable results to $100 \%$. Reducing the proportion of incoming instances provides a significant reduction on the time and resource (people) required to perform the labelling task and the update of the model.

2) Weighting on Newest Samples: In this experiment, we have updated the model using only $50 \%$ of the incoming data. On top of that, we have given more importance to the new incoming data through weighting.

Fig 5 shows how the model has performed after applying weights on top of the 50\% random instances. The thicker (blue) line represents the model retrained with $50 \%$ random instances without weight, whereas the thin line represent the model retrained with the same $50 \%$ random instances, with different proportion of weights on them $(2: 1,3: 1,5: 1$ and 10:1).
Visually, we see that the models with weighting schemes have achieved in $w 4$ the best performance compared to the baseline (thicker line). Although, in few points of D1 and D4 it was observed a decreasing in performance for some weights.

\section{DEGREE OF DRIFT OF DYNAMIC MODELS}

According to [14] and [15], there are four common drift patterns: Sudden, Incremental, Gradual and Reoccurring. We have noticed that the drift in D1 and D4 occurs gradually, where D2 the models stays practically flat. In order to measure this drift, we have calculated the slope of the line in dataset D1, D2 and D4 throughout a 1-year period. Negative values mean that the performance of the model has a tendency to decrease, whereas positive values is the opposite. In Table $\mathrm{V}$, we show a comparison between the static (baseline) and dynamic model after applying different values of weight on $50 \%$ random samples.

It is observed that by retraining the model, the performance over time is improved over the baseline static model. With a minimum of $50 \%$ of retraining of the incoming messages we achieve such improvement. This result has shown that we can use only $50 \%$ of the incoming instances to retrain the model and still improves over the static model. Thus, reducing workload of labellers in $50 \%$.

In addition, by weighting the newest incoming samples, we have achieved further improvements. In Table V, if we consider the average results, the best configuration for avoiding performance deterioration over time is by retraining $50 \%$ of new incoming instances and adding weights on them with a 3:1 proportion.

TABLE V: SLOPE OF THE LINE FOR STATIC AND DYNAMIC MODELS

\begin{tabular}{l|ccc|c} 
& D1 & D2 & D4 & Avg \\
\hline Static. (Baseline) & -.020 & +.005 & -.019 & -.011 \\
\hline \hline Dyn. (50\% relabeled) & +.001 & $\mathbf{+ . 0 1 5}$ & +.003 & .005 \\
Dyn. (50\% relabeled + x2 weight) & $\mathbf{+ . 0 0 8}$ & +.019 & +.003 & .010 \\
Dyn. (50\% relabeled + x3 weight) & +.005 & +.017 & $\mathbf{+ . 0 1 2}$ & $\mathbf{. 0 1 1}$ \\
Dyn. (50\% relabeled + x5 weight) & -.001 & $\mathbf{+ . 0 2 2}$ & +.008 & .009 \\
Dyn. (50\% relabeled + x10 weight) & .000 & $\mathbf{+ . 0 2 2}$ & +.007 & .009 \\
\hline
\end{tabular}



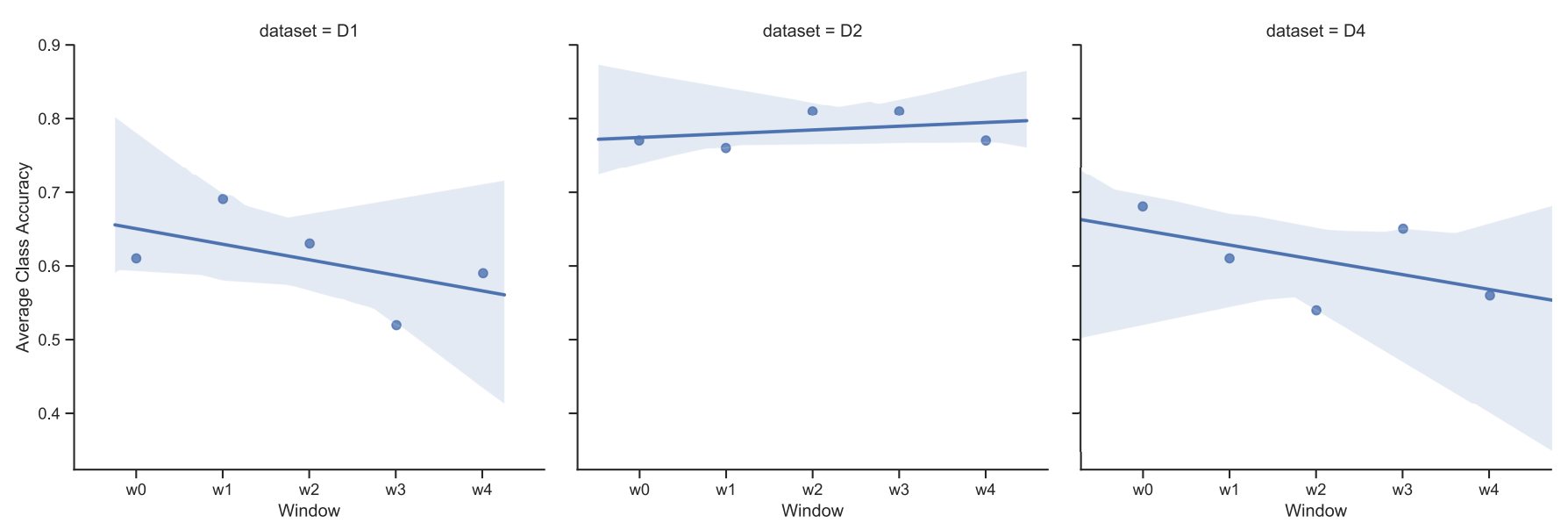

Fig. 2: Static Model (baseline) - Average Class Accuracy results of the model trained in 4 months period ( $w 0)$ and tested in every two months $(w 1 \ldots w 4)$ in 1-year period

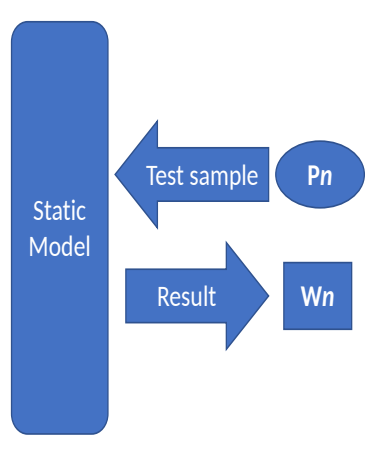

(a) Static Model

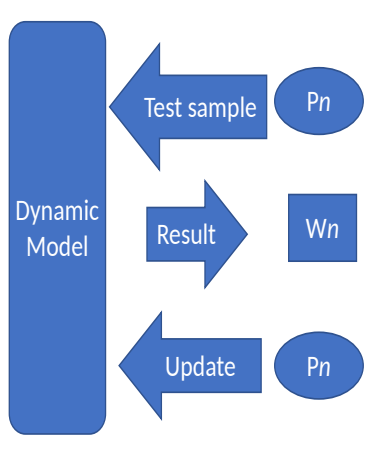

(b) Dynamic Model

Fig. 3: Experimental procedure, where $\mathrm{P}=$ sample, $\mathrm{W}=$ result in Avg acc. and $n=$ period

\section{CONCLUSiOn AND Future Work}

In this paper, we have investigated how concept drift affects the accuracy of machine learning models applied to detection of software vulnerability communication in social media and hacker forums. As the nature of computer security and language are dynamic, we confirm our expectation that the performance of these models decreases with the rapidly changing of concept within a 1-year period. However, models trained and applied to Twitter messages do not show apparent drift. We believe that, compared to other hacker forums, Twitter provides shorter and less variable post content with regards to novelty on hacker techniques and exploitation tools. Thus, we imply that the drift on this platform is not enough to affect the performance of such models in 1-year period. Moreover, its users (even security researchers) use a more direct style of communication to reach broad audience without the need to deepen into technical details with specific and novel terms.

Furthermore, in order to provide a solution to the drift problem, we have investigated techniques such as periodic

update and weighting. We have found that by updating the model with $50 \%$ of the incoming instances in a bimester while giving them weights in a proportion of 3:1 is sufficient to avoid the performance decreasing.

We acknowledge that, in real-life situation, it still infeasible to perform the labelling of 50\% of messages in larger streams of data. However, with these results we highlight two important findings: (1) the importance of retraining the model over new (recent) messages and weight them to maintain the performance, and (2) not all streamed messages have the same importance, as the drift can be avoided by at least $50 \%$ of the relabelled data. We also point out that our experiment is limited to the amount of data we collected. Further tests need to be done with great volumes of posts in longer periods of time.

As future work, we intend to study the characteristics (patterns) of the worth-to-label messages in the data stream. Finding these messages would allow us to reduce the labelling task workload while maintaining the model usable for longer periods. We are also investigating concept-drift-detection algorithms, such as ADAWIN [9], EWMA [10] to trigger the labelling task after a potential change into the underlying distribution of the incoming data.

\section{ACKNOWLEDGMENT}

Andrei Lima Queiroz would like to thank the scholarship granted by the Brazilian Federal Programme Science without Borders supported by CNPq (Conselho Nacional de Desenvolvimento Científico e Tecnológico), No 201898/2015-2. 

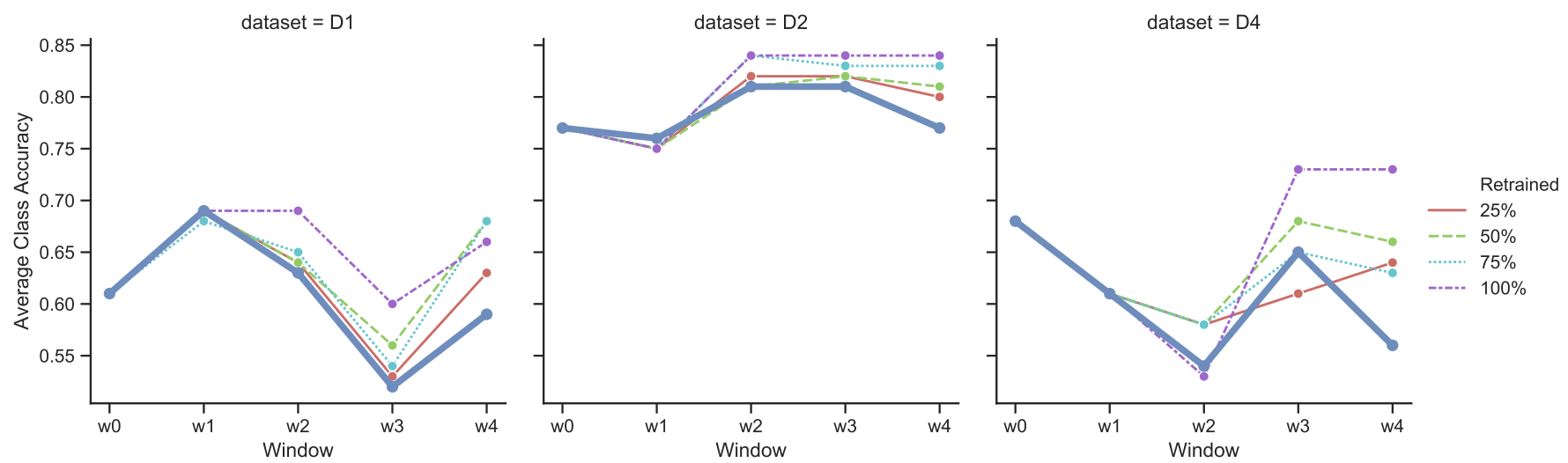

Fig. 4: Comparing the baseline static model, bold line (blue), against models retrained with different proportions $(25,50,75$, $100) \%$ of past instances
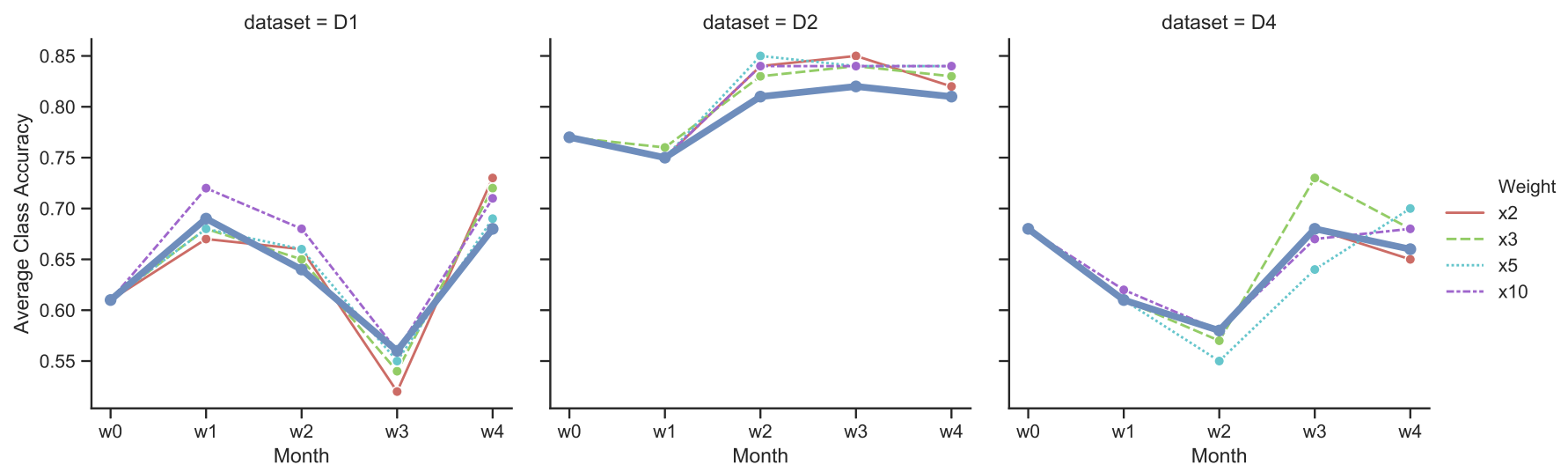

Fig. 5: Comparing models with different values of weights on new instances (x2, x3, x5, x10) against model with no weights, bold line (blue). All models using 50\% proportion of incoming instances

\section{REFERENCES}

[1] S. Greengard, "Cybersecurity gets smart," Communications of the ACM, vol. 59, no. 5, pp. 29-31, 2016.

[2] T. S. Sethi and M. Kantardzic, "When good machine learning leads to bad security: Big data (ubiquity symposium)," Ubiquity, vol. 2018, no. May, May 2018. [Online]. Available: https://doi.org/10.1145/3158346

[3] K. Zhao, Y. Zhang, C. Xing, W. Li, and H. Chen, "Chinese underground market jargon analysis based on unsupervised learning," in 2016 IEEE Conference on Intelligence and Security Informatics (ISI), 2016, pp. $97-$ 102.

[4] S. Chatterjee and S. Thekdi, "An iterative learning and inference approach to managing dynamic cyber vulnerabilities of complex systems," vol. 193, 2020, p. 106664.

[5] S. Hilt, V. Kropotov, F. Merces, M. Rosario, and D. Sancho, "The internet of things in the cybercrime underground," uRL: https://media.rbcdn.ru/media/reports/ wp-the-internet-of-things-in-the-cybercrime-underground.pdf [Accessed: Jan, 2020].

[6] R. P. Lippmann, W. M. Campbell, D. J. Weller-Fahy, A. C. Mensch, G. M. Zeno, and J. P. Campbell, "Finding malicious cyber discussions in social media," Lincoln Laboratory Journal, vol. 22, no. 1, pp. 46-59, 2016.

[7] I. Deliu, C. Leichter, and K. Franke, "Extracting cyber threat intelligence from hacker forums: Support vector machines versus convolutional neural networks," in 2017 IEEE International Conference on Big Data (Big Data), Dec 2017, pp. 3648-3656.

[8] R. Jordaney, K. Sharad, S. K. Dash, Z. Wang, D. Papini, I. Nouretdinov, and L. Cavallaro, "Transcend: Detecting concept drift in malware classification models," in 26th USENIX Security Symposium (USENIX Security 17). Vancouver, BC: USENIX Association, Aug. 2017, pp. 625-642. [Online]. Available: https://www.usenix.org/conference/ usenixsecurity $17 /$ technical-sessions/presentation/jordaney

[9] A. Bifet and R. Gavaldà, "Adaptive learning from evolving data streams," in Advances in Intelligent Data Analysis VIII, N. M. Adams, C. Robardet, A. Siebes, and J.-F. Boulicaut, Eds. Berlin, Heidelberg: Springer Berlin Heidelberg, 2009, pp. 249-260.

[10] G. J. Ross, N. M. Adams, D. K. Tasoulis, and D. J. Hand, "Exponentially weighted moving average charts for detecting concept drift,” Pattern Recogn. Lett., vol. 33, no. 2, p. 191198, Jan. 2012. [Online]. Available: https://doi.org/10.1016/j.patrec.2011.08.019

[11] A. L. Queiroz, S. Mckeever, and B. Keegan, "Detecting hacker threats: Performance of word and sentence embedding models in identifying hacker communications," in The 27th AIAI Irish Conference on Artificial Intelligence and Cognitive Science, 2019, p. in press.

[12] _ _ "Eavesdropping hackers: Detecting software vulnerability communication on social media using text mining," in The Fourth International Conference on Cyber-Technologies and Cyber-Systems, 2019, pp. 41-48.

[13] J. I. G. Hidalgo, B. I. F. Maciel, and R. S. M. Barros, "Experimenting with prequential variations for data stream learning evaluation," vol. 35, no. 4, 2019, pp. 670-692. [Online]. Available: https://onlinelibrary.wiley.com/doi/abs/10.1111/coin.12208

[14] I. Zliobaite, M. Pechenizkiy, and J. Gama, An Overview of Concept Drift Applications, ser. Studies in Big Data. Switzerland: Springer International Publishing AG, 2016, pp. 91-114.

[15] S. V. Kadam, "A survey on classification of concept drift with stream data," 2019, uRL: https://hal.archives-ouvertes.fr/hal-02062610 [Accessed: Jan, 2020]. 